critical to mouse development. Nat Genet 2001 ; 28 : 241-9.

11. Leighton PA, Mitchell KJ, Goodrich LV, et al. Defining brain wiring patterns and mechanisms through gene trapping in mice. Nature $2001 ; 410$ : 174-9.

12. Tordjman R, Ortega N,
Coulombel L, Plouet J, Romeo PH, Lemarchandel V. Neuropilin-1 is expressed on bone marrow stromal cells: a novel interaction with hematopoietic cells? Blood 1999 ; 94 : 2301-9.

13. Pinson KI, Brennan J, Monkley S, Avery BJ,
Skarnes WC. An LDLreceptor-related protein mediates Wnt signalling in mice. Nature 2000 ; 407 : 535-8.

14. Burgess RW, Skarnes WC, Sanes JR. Agrin isoforms with distinct amino termini: differential expression, localization, and function. J Cell Biol 2000 ; $151: 41-52$.

15. Wempe F, Yang JY, Hammann J, Melchner H. Gene trapping identifies transiently induced survival genes during programmed cell death. Genome Biol $2001 ; 2: 1-10$.

\title{
NOUVELLE
}

\section{La relaxine : une ancienne hormone trouve enfin ses récepteurs}

Nicolas Chartrel, Hubert Vaudry
Inserm U.413, Institut

Fédératif de Recherches Multidisciplinaires Université de Rouen, 76821 Mont-Saint-Aignan Cedex, France. sur les Peptides $n^{\circ} 23$,

montré que la protéine joue un rôle crucial dans l'induction de la phase de décidualisation [6]. La relaxine, qui favorise aussi l'expression du facteur de croissance endothélial
$>$ L'histoire de la relaxine commence il y a 75 ans lorsque Frederick Hisaw, en étudiant les activités hormonales du corps jaune du follicule ovarien, identifie deux principes actifs, la « corporine », qui induit des transformations de type progestatif dans l'utérus, et la « relaxine » qui provoque un relâchement de la symphyse pubienne [1]. Ce n'est que 50 ans plus tard que la structure de la molécule responsable de l'activité myorelaxante a pu être élucidée [2]. La relaxine est une hormone polypeptidique de structure hétérodimérique appartenant à la superfamille de l'insuline (Figure 1) qui comprend également les insulin-like growth factor-I et -II (IGF-I et $-I I$ ) et le relaxin-like factor (RLF ou INSL3). Chez la femme, la relaxine est essentiellement produite par le corps jaune alors que chez l'homme, la principale source de relaxine réside dans la prostate $[3,4]$. Chez de nombreuses espèces de mammifères comme le porc, le rat ou le cobaye, la relaxine joue un rôle essentiel dans la croissance et le remodelage des organes génitaux pendant la gestation et plus particulièrement au moment de la parturition, en provoquant l'élongation des ligaments pelviens et le relâchement du col de l'utérus [5]. De plus, chez le rat, la relaxine inhibe les contractions spontanées ou induites par l'ocytocine du myomètre utérin. Cependant, chez la femme, la relaxine n'exerce aucune activité sur la relaxation du col de l'utérus pendant l'accouchement. En revanche, dans l'espèce humaine, un pic de relaxine circulante est observé en début de grossesse, au moment de la nidification de l'embryon, et il a été dans l'endomètre utérin, est directement impliquée dans le développement de nouveaux vaisseaux sanguins indispensables à la croissance de l'embryon [7]. Par ailleurs, la relaxine, qui est produite par de nombreux tissus autres que ceux constituant les organes génitaux, n'est pas uniquement une hormone de la grossesse : elle est notamment responsable de la néoangiogenèse après un infarctus ou pendant

\begin{tabular}{|r|c|c|c|c|c|}
\hline Pré-pro-insuline & PS & Chaîne B & \multicolumn{2}{c}{ Peptide C } & Chaîne A \\
Pré-pro-relaxine & PS & Chaîne B & \multicolumn{2}{c}{ Peptide C } & Chaîne A \\
Pré-pro-RLF & PS & Chaîne B & Peptide C & Chaîne A & \\
Pré-pro-IGF-I & PS & Chaîne B & C Chaîne A & D & Peptide E \\
Pré-pro-IGF-II & PS & Chaîne B & C Chaîne A D & Deptide E \\
\hline
\end{tabular}

Figure 1. Structure des précurseurs des polypeptides de la superfamille de l'insuline. Toutes les prohormones de cette famille possèdent un peptide $C$ de connexion qui favorise la formation de deux ponts disulfures entre les chaînes $A$ et $B$ de l'hormone mature. Dans la proinsuline, la prorelaxine et le pro-RLF, les chaînes $A$ et $B$ sont respectivement localisées dans les régions $C$ - et $N$ terminales du précurseur et sont séparées par un peptide $C$ de grande taille qui est excisé au cours de la maturation de l'hormone active. En revanche, pour les pro-IGF-I et -II, le peptide C est de petite taille et il reste présent dans I'hormone mature. Les pro-IGF-I et -II contiennent également dans leur région $C$-terminale deux autres fragments nommés peptides $D$ et $\varepsilon$, le peptide $\varepsilon$ étant excisé au cours de la maturation de l'hormone. PS : peptide signal. 
une cicatrisation, et elle prévient l'apparition de fibrose.

Alors que la relaxine était l'une des toutes premières hormones peptidiques impliquées dans la reproduction à avoir été identifiée, son récepteur n'avait toujours pas été caractérisé. Cette lacune vient d'être comblée par l'équipe de Aaron Hsueh qui montre que la relaxine est le ligand naturel de deux récepteurs à 7 domaines transmembranaires couplés aux protéines $G$ (RCPG) orphelins, les LGR7 et LGR8 [8]. Ce travail démontre une nouvelle fois la pertinence de la stratégie de pharmacologie inverse, laquelle consiste à identifier le ligand d'un RCPG orphelin à partir de fractions purifiées de tissus ou d'une librairie de molécules synthétiques. Toutefois, la démarche suivie par les chercheurs de l'Université de Stanford diffère assez sensiblement de l'approche classique de pharmacologie inverse telle qu'elle a déjà été décrite dans les colonnes de médecine/sciences [9-11] et mérite donc qu'on s'y arrête.

Ces chercheurs ont tout d'abord noté que les souris dont le gène du RLF a été invalidé présentent une anomalie de descente des testicules identique à celle qui est observée chez des souris dépourvues du gène GREAT qui code pour un GPCR orphe-

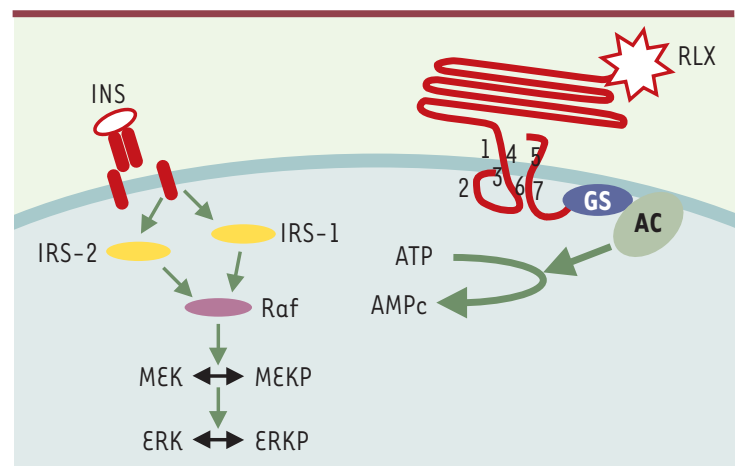

Figure 2. Comparaison des mécanismes d'action de l'insuline et de la relaxine. Bien que les deux hormones appartiennent à la même famille structurale, leurs récepteurs sont totalement différents. L'insuline (INS) agit via un récepteur tyrosinekinase dont l'autophosphorylation va activer la cascade de kinases Raf/MEK/ERK. En revanche, la relaxine (RLX) active l'adénylyl-cyclase $(A C)$ par l'intermédiaire d'un récepteur à 7 domaines transmembranaires couplé à une protéine G (GS). IRS- 1 et -2 : insulin receptor substrate- 1 et -2 . lin $[12,13]$. Ce premier constat les a conduits à émettre l'hypothèse que des polypeptides de type relaxine pouvaient être des ligands de GPCR et non pas de récepteurs membranaires de type tyrosine-kinase comme leur appartenance à la superfamille de l'insuline le suggérait (Figure 2). Le fait que la relaxine provoque un accroissement des concentrations intracellulaires en AMPc dans la plupart de ses tissus cibles, et notamment dans les cellules endométriales, confortait cette hypothèse. Parallèlement, cette même équipe avait identifié deux nouveaux RCPG, le LGR6 et le LGR7, dont la structure est apparentée à celle des récepteurs de la LH et de la FSH [14], et la recherche de paralogues du LGR7 dans le génome humain avait amené les auteurs à identifier un nouveau récepteur orphelin, le LGR8, qui s'est avéré être l'orthologue du récepteur GREAT murin. L'ensemble de ces observations indiquait que la relaxine pouvait être un ligand potentiel du LGR7 et/ou du LGR8.

Afin de tester cette hypothèse, Aaron Hsueh et ses collaborateurs ont transfecté des cellules 293 T avec l'ADNc codant pour le LGR7 et le LGR8 [14]. Ils ont montré que la relaxine provoque une stimulation dépendante de la dose de la concentration intracellulaire d'AMPc à la fois dans des cellules surexprimant le LGR7 et celles surexprimant le LGR8, avec des $\varepsilon C_{50}$ respectifs de 1,5 et $5 \mathrm{nM}$ alors que I'insuline, I'IGF-I, I'IGF-II et le glucagon n'ont aucun effet. Ils ont aussi mis en évidence des différences dans l'expression tissulaire du LGR7 et du LGR8 : les transcrits du LGR7 sont fortement exprimés dans le cerveau, l'ovaire, le testicule, l'utérus, la prostate et le cœur, alors que ceux du LGR8 sont principalement détectés dans le cerveau, les muscles, le testicule, l'utérus, la thyroïde et la moelle osseuse. L'utilisation d'anticorps dirigés spécifiquement contre le LGR7 a révélé que, chez la ratte, le récepteur est surtout présent dans le myomètre de l'utérus et dans le vagin. Ces différentes données pharmacologiques et anatomiques suggèrent que la majorité des activités biologiques de la relaxine s'exercent par l'intermédiaire $d u$ LGR7. Par ailleurs, le phénotype commun des souris déficientes en RLF et en récepteurs GREAT, ainsi que la forte homologie observée entre le récepteur GREAT et le LGR8 font penser que le LRF pourrait être un ligand naturel du LGR8.

Dans cette même étude, les auteurs ont produit une protéine de fusion constituée du domaine ectoplasmique du récepteur LGR7 et de la région transmembranaire de l'antigène CD8 des lymphocytes T. Après traitement des cellules à la thrombine, cette protéine chimère libère l'ectodomaine du LGR7, dénommé 7BP, qui possède une bonne capacité de liaison de la relaxine. En particulier, l'administration par voie sous-cutanée de 7BP à des souris pendant les quatre derniers jours de la gestation retarde de 27 heures le moment de la parturition. La protéine 7BP se comporte donc comme un antagoniste fonctionnel de la relaxine, à l'instar du domaine $\mathrm{N}$-terminal des récepteurs des hormones gonadotropes et thyréotropes vis-à-vis de la LH, de la FSH et de la TSH [15].

La découverte des récepteurs de la relaxine constitue une avancée importante tant sur le plan fondamental que pour ses applications physiopathologiques éventuelles. La forte identité de structure du LGR7 et du LGR8 avec les récepteurs des hormones gonadotropes suggère que ces récepteurs ont une origine phylogénétique commune. La recherche de récepteurs orphelins paralogues des récepteurs de la $\mathrm{LH}$, de la FSH et de la relaxine pourrait ainsi permettre d'identifier de nouveaux facteurs peptidiques de régulation de la fonction de reproduction. La caractérisation des récepteurs de la relaxine devrait également conduire, à terme, au développement d'agonistes et antagonistes stables de l'hormone, utilisables à des fins 
thérapeutiques. On peut en effet rappeler que, chez la femme ménopausée, la chute des hormones stéroïdes sexuelles s'accompagne d'une diminution importante des taux de relaxine. Compte tenu de certains symptômes associés à la ménopause tels que la prédisposition au développement de fibroses, les défauts de cicatrisation ou les troubles vasculaires, une hormonothérapie substitutive à base d'agonistes de la relaxine pourrait offrir des perspectives intéressantes. $\Delta$

The receptor for relaxin has finally been identified

\section{RÉFÉRENCES}

1. Hisaw FL. Experimental relaxation of the pelvic ligament of the guinea pig. Proc Soc Exp Biol Med 1926 ; 23: 661-3.

2. Schwabe C, McDonald JK. Primary structure of the $B$ chain of porcine relaxin. Biochem Biophys Res
Commun 1977 ; 75 : 503-10.

3. Weiss G, O’Byrne EM, Steinetz BG. Relaxin : a product of the human corpus luteum of pregnancy. Science 1976 ; 194 : 948-9.

4. Ivell R, Hunt N, KhanDawood F, Dawood My. Expression of the human relaxin gene in the corpus luteum of the menstrual cycle and in the prostate. Mol Cell Endocrinol 1989 ; $66: 251-5$

5. Sherwood OD. Relaxin. In : Physiology of reproduction, $2^{\text {nd }}$ ed. New York : Raven press, 1994 : 861-1009.

6. Tellgmann R, Gellersen B. Marker genes of decidualization : activation of the decidual prolactin gene. Hum Reprod Update 1998 ; 4 : 472-9.

7. Unemori $\varepsilon N$, Erikson $M \varepsilon$, Rocco SE, et al. Relaxin stimulates expression of vascular endothelial growth factor in normal human endometrial cells in vitro and is associated with menometrorrhagia in women. Hum Reprod 1999 ; $14: 800-6$.

8. Hsu Sy, Nakabayashi K, Nishi $\mathrm{S}$, et al. Activation of orphan receptors by the hormone relaxin. Science 2002 ; 295 : 671-4.

9. Vaudry H, Braun B, Chartrel N. La pharmacologie inverse marque des points : découverte d'un nouveau peptide stimulant la sécrétion de prolactine. Med Sci 1998 ; 14 : 1118-20.

10. Vaudry H, Anouar Y, Galas L, Tonon MC, Chartrel N, Llorens-Cortes C. La ghréline, un nouveau neuropeptide stimulant la sécrétion de l'hormone de croissance. Med Sci 2000 ; $16: 555-7$.

11. Vaudry $\mathrm{H}$, Coulouarn $\mathrm{y}$, Lihrmann I, et al. Deux neuropeptides orphelins trouvent enfin leur récepteur. Med Sci 2000 ;

$16: 426-9$.

12. Nef S, Parada LF.

Cryptorchidism in mice mutant for INSL3. Nat Genet 1999 ; 22 : 295-9.

13. Overbeek PA, Gorlov IP, Sutherland RW, et al. A transgenic insertion causing cryptorchidism in mice. Genesis 2001 ; 30 : 26-35.

14. Hsu Sy, Kudo M, Chen T, et al. The three subfamilies of leucin-rich repeatcontaining $\mathrm{G}$ proteincoupled receptors (LGR): identification of LGR6 and LGR7 and the signalling mechanism for LGR7. Mol Endocrinol 2000 ; 14 : 1257-71.

15. Osuga $Y$, Kudo M, Kaipia A, Kobilka B, Hsueh AJ. Derivation of functional antagonists using $\mathrm{N}$ terminal extracellular domain of gonadotropin and thyrotropin receptors. Mol Endocrinol 1997 ; 11 : 1659-68.

\section{NOUVELle}

Pep- 1 transporte des protéines
dans les cellules
de mammifères
May Catherine Morris, Frédéric Heitz, Gilles Divita

$>$ Au cours des dix dernières années, la recherche dans le domaine de la thérapie génique a été principalement focalisée sur l'amélioration des vecteurs viraux et non viraux pour le transfert de gènes. Toutefois, les formulations proposées pour l'introduction de « transgènes » restent encore limitées en raison de difficultés liées à leur pouvoir immunogène, à leur toxicité et à leur faible efficacité in vivo. Face à ces limitations, l'alternative était d'imaginer de nouvelles stratégies qui permettraient le transfert direct de la protéine d'intérêt dans un grand nombre de cellules [1]. Le développement de peptides ou pro- téines à visée thérapeutique reste limité par la faible perméabilité membranaire et par la présence, à la surface de la cellule, de protéines ou de récepteurs qui contrôlent sélectivement le transport cellulaire de molécules. Récemment, ces restrictions ont été levées par la découverte de petits domaines protéiques, appelés protein transduction domains (PTD). Ces derniers, de nature basique, sont capables de traverser les membranes biologiques indépendamment de la présence de transporteurs ou de récepteurs spécifiques à la surface des cellules, et permettent le transfert de peptides ou de protéines dans
Centre de Recherche en Biochimie

Macromoléculaire (CRBM),

Cnrs UPR 1086,

1919 , route de Mende,

34293 Montpellier, France.

divita@crbm.cnrs-mop.fr

les cellules [1, 2]. Les trois domaines PTD qui ont été le mieux caractérisés et utilisés dérivent de la protéine Tat du virus de l'immunodéficience humaine (VIH-1) [3], de la troisième hélice de l'homéo-domaine d'Antennapedia (Antp) [4], et de la protéine de structure Vp22 du virus de l'herpès simplex [5]. À ce jour, le PTD de la protéine Tat constitue le système le plus efficace pour le transfert des protéines in vitro et in vivo $[6,7]$. Cependant, I'utilisation des vecteurs PTD présente un certain nombre de contraintes réduisant leur exploitation 